\title{
Balancing Privacy and Utility with Pattern Based Activity Detection
}

\author{
Extended Abstract \\ Cassandra Carley \\ Duke University \\ carley@cs.duke.edu
}

\begin{abstract}
The diffusion of surveillance cameras often leads to conflicts between utility, that is, the benefits of preserving the information the camera records, and privacy, that is, the ability for the people being observed to conceal information they want to protect. For example, a camera monitoring an office kitchen may be useful in identifying a food thief, but might unintentionally reveal the PIN someone enters on a mobile phone. We design a video processing system that detects private activities in surveillance video and filters them out of the recording with minimal disruption of video quality. At the core of our system is the light-weight computation of a fixed-size feature that describes the spatio-temporal aspects of human activities that extend over variable amounts of time and space. Converting events of variable length and extent to a fixed-size descriptor makes it possible to use off-the-shelf classifiers to recognize and localize activities to be protected from recording. Comparisons of our descriptor with several alternatives show improved performance with less computation. We contribute two new video datasets recorded with a kitchen security camera, and we carry out a pilot user study to show that PIN theft is a valid concern.
\end{abstract}

\section{CCS CONCEPTS}

- Security and privacy $\rightarrow$ Human and societal aspects of security and privacy; Privacy protections; Usability in security and privacy;

\section{KEYWORDS}

Activity detection; Video analysis; Hand tracking

\section{ACM Reference Format:}

Cassandra Carley. 2018. Balancing Privacy and Utility with Pattern Based Activity Detection: Extended Abstract. In 2018 AAAI/ACM Conference on AI, Ethics, and Society (AIES '18), February 2-3, 2018, New Orleans, LA, USA. ACM, New York, NY, USA, 2 pages. https://doi.org/10.1145/3278721.3278800

\section{FRAMEWORK}

Nowadays, video is being collected for public and private uses at locations including our homes, offices, airports, malls, and other public spaces. Cameras are either installed at fixed locations, mounted on vehicles, or body-worn. While collected video serves some purpose, such as catching a thief, identifying a person, or tracking

Permission to make digital or hard copies of part or all of this work for personal or classroom use is granted without fee provided that copies are not made or distributed for profit or commercial advantage and that copies bear this notice and the full citation on the first page. Copyrights for third-party components of this work must be honored.

For all other uses, contact the owner/author(s).

AIES '18, February 2-3, 2018, New Orleans, LA, USA

(c) 2018 Copyright held by the owner/author(s).

ACM ISBN 978-1-4503-6012-8/18/02.

https://doi.org/10.1145/3278721.3278800 someone's health, it also often contains aspects of behavior that are irrelevant to the application and needlessly infringe on a person's privacy. These aspects include the identity of people in the background or any text someone may be typing on a cellphone. A fundamental tension then arises between extracting useful information from videos and protecting privacy.

We propose a video-processing framework to resolve this tension. Our pipeline feeds frame-level descriptors to a classifier and applies a suitable privacy filter that pixelates or otherwise hides the information to be protected, while keeping the video useful for its intended purpose. The main role of the descriptor is to convert patterns of activity that extend over indeterminate amounts of time and over irregular regions of image space into a vector of fixed length. As a result of this conversion, any of a number of classifiers can be used to detect whether a privacy-sensitive activity is taking place. In particular, we use convolutional neural networks, cognizant of the recent successes of these architectures.

\section{LIGHTWEIGHT TRAJECTORY DESCRIPTOR}

The traditional computer vision approach to activity detection is essentially generative: If the activity involves someone's hand, as it does in our initial experiments, this approach would involve designing a model of the hands shapes and their skeletons, and estimating skeletal motions from segmented image frames. This approach is both daunting and unnecessary. First, hands move quickly, making tracking problematic. Second, finding the hand in the image is a difficult segmentation problem. Third, the interaction of hands and the objects they manipulate (such as a cell phone) causes occlusions and requires further modeling and estimation. All this reconstruction is overkill if the goal is the classification of activities into private and not. Instead, we bypass the generative approach by designing a fixed-length descriptor of activity in video that is estimated through a lightweight computation. The descriptor is then fed to a classifier that answers the question in a discriminative fashion.

Our descriptor treats activity as a sort of motion texture, akin to the representations used in the literature to describe natural processes such as smoke and waves. The presence of an object being manipulated (cellphone) affects this texture in informative ways, rather than making estimation harder, and a liability thereby becomes an asset. To implement this idea, we first compute trajectories $\mathbf{p}(t)$ by detecting salient points in the video and tracking them over time. We then base the temporal aspects of our descriptor on the notion of auto-covariance:

$$
\mathbb{E}[\mathbf{q}(t) \mathbf{q}(t+\tau)] \text { where } \mathbf{q}(t)=\mathbf{p}(t)-\mathbb{E}[\mathbf{p}(t)]
$$


In this way, the number of auto-covariance values per point is the number of time lags $\tau$, a fixed design parameter. To capture the spatial aspects, we introduce a variant of the cell based representations used in HOG (Histogram of Oriented Gradient) [1] or SIFT (Shift-Invariant Feature Transform) [2] methods. In our descriptor, each cell contributes a vector of auto-covariance values, one vector entry per time lag.

\subsection{Privacy Filter}

A descriptor for each frame is fed to a classifier, and image regions deemed private are concealed. In a first implementation, we black out the entire frame. However, we plan to devise concealment methods that preserve the utility of the non-offending parts of the video as much as possible. These methods include blurring or pixelating near the fingertips. We intend to explore further options to achieve a good balance between privacy and utility.

\section{TRAINING AND EVALUATION}

We created an annotated data set for both training and evaluation of our system. The video in the data set was taken in an office kitchen, with a GoPro Hero 4 Session camera we placed next to an actual surveillance camera. While the surveillance system is meant to identify food thieves or vandals, it should not allow intruding on the privacy of the kitchen users. In particular, it should not be possible to discern anything anyone types on their cell phones while they wait for their coffee to brew. With these considerations in mind, we make the following contributions:

\subsection{Training Data}

Effectively training the classifier requires samples for both positive and negative examples of our target activity, typing on a cell phone. For positive samples, we must address different variations in activity including hand shape, motion speed, viewpoint, and phone appearance and setting. For negative samples, we include sequences where hands are not present, hands are not the focus, phones are not present, the phone is occluded by the hand, hands that interact with the phone in ways other than typing, and hands that are present but engaged in non-phone activities. These include holding the phone without typing, getting the phone out of the pocket, and opening the fridge. To achieve better generalization we use many negative samples of hands interacting and freely moving in proximity to a cell phone and several other kitchen and deskrelated objects. In total, we used 3 different users (authors of the work and consenting collaborators) and 15 different PINs to capture 18 different video sequences, totaling 87,973 frames or 24.4 minutes of footage at $60 \mathrm{fps}$. Each frame was annotated with a detailed label corresponding to the activity which was used to create positive and negative samples given the desired target activity (PIN entry).

\subsection{Evaluation}

We use our data set to plot precision-recall curves for activity classification, and to evaluate the robustness of our approach to variations in hand shape, typing speed, viewpoint, and settings. We compare our descriptor (AC) with several baseline descriptors, including raw frames, frame differences, and point trajectories. Our aim is to showcase the advantages of spatial aggregation and the effectiveness of our fixed-size descriptor in encoding temporal information over varying time intervals. Preliminary results show our descriptor achieves superior results to the best baseline (frame differences) with a smaller footprint and less training time (Figure 1).

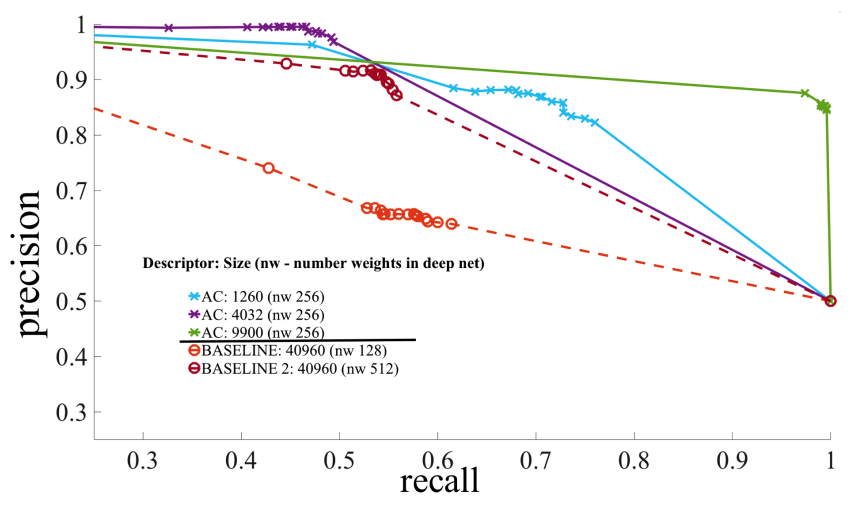

Figure 1: Results show improvement of AC descriptors (across several sizes) over the best BASELINEs for detecting PIN entry. Descriptors consider a 100 frame history. BASELINEs use images down-sampled to $64 \times 64$ pixels and sampled at every 10 frames. Increasing the number of weights (nw) in the deep net increases the precision at minimal cost to recall, but AC still outperforms BASELINE. Experiment uses 10, 000 training samples and 1,000 testing samples with an even split between positive and negative labels.

\section{SUMMARY AND FUTURE WORK}

We propose a system for the protection of private information in surveillance video, based on activity classification and an unobtrusive filter. A fixed-length descriptor of activities with variable temporal length and spatial extent allows using off-the-shelf classifiers, including convolutional neural nets. Preliminary experiments show promising precision, recall, and computational efficiency.

In future work, we plan to improve activity localization in the image for minimal obtrusion, refine our descriptor for maximal discriminative ability, enlarge our training and evaluation data sets, and extend our demonstrations to other types of activities, including those that do not involve hands.

\section{ACKNOWLEDGMENTS}

This material is based upon work supported by the NSF under Grants No. IIS-1208245 and CCF-1513816 and in collaboration with Nisarg Raval and Carlo Tomasi.

\section{REFERENCES}

[1] Navneet Dalal, Bill Triggs, and Cordelia Schmid. 2006. Human detection using oriented histograms of flow and appearance. In European conference on computer vision. Springer, 428-441.

[2] David G Lowe. 2004. Distinctive image features from scale-invariant keypoints. International journal of computer vision 60, 2 (2004), 91-110. 